\title{
232. Sentence-oriented semantic approaches in generative grammar
}

1. Introduction

2. A generative grammar as an algorithm

3. The semantic component

4. Bibliography

\section{Introduction}

Throughout the 20th century up to the present day grammar and semantics have been uneasy bedfellows. A look at the historical background will make it clear how this curious situation came about. 20th-century linguistics has been characterized by an almost exclusive concern with the structure of words, word groups and sentences. This concern was reinforced, especially on the American side of the Atlantic, by the sudden rise and subsequent dominance of behaviorism during the 1920s. It started in psychology but quickly permeated all the human sciences, including linguistics, until the early 1960s, when it collapsed as suddenly as it had arisen.

Behaviorism was based on three premises. First, it stated the principle that all theories about the workings of whatever it is that one may call the mind should be based on and motivated by observable data drawn from the ways in which the organisms said to be equipped with a mind behave. Reliance on, or reports of, private experiences are not data in this sense and must be left out of account.

Secondly, while many forms of behavior are directly reducible to physical causes, many other forms of behavior are not, or at least appear not to be. For example, passing out after a heavy blow on the head is a form of behavior that has a clear physical cause. But getting red in the face after being caught out on a lie is not so obviously reducible to a physical cause. Those forms of behavior that cannot be attributed to direct physical causes form the subject of psychology, which deals with the question of what causes these forms of behavior.

Thirdly, when posing the question of the causality of the latter forms of behavior, psychology should keep to the simplest possible hypothesis. The behaviorist hypothesis (inspired by experiments on animal behavior, such as salivating in the sight of food) was that the forms of behavior studied in psychology are likewise the result of physical causation, but indirectly, via an intervening mechanism of associations of sensory stimuli. Present-day psychology, in particular the part known as cognitive science, accepts the first two premises but denies the third, in that nowadays the minimal hypothesis of stimulus association is considered far too restricted to explain the enormous variety of human behavior. Instead, it is now currently assumed that the bulk of human behavior is the result of complex computational processes carried out by the brain, mostly below any threshold of awareness. It is even widely, but not universally, assumed that what used to be called 'the mind' should, in principle, be reducible to a set of computationial mechanisms in the brain. In the context of behaviorism it thus became highly unfashionable, if not downright anathema, to talk about the mind in any of its manifestations, including cognition.

In linguistics, this created an atmosphere in which anything to do with meaning was looked at with suspicion. One was willing to accept that something like 'meaning' is probably there in language, but it was not considered to be a legitimate object of scientific investigation. A truly scientific linguistics should look exclusively at the forms occurring in language and language use, and disregard meaning altogether.

\section{A generative grammar as an algorithm}

In this climate, a grammar of a language $\mathbf{L}$ came to be seen as the set of rules and principles that define all and only the well-formed combinations of the morphemes of $\mathbf{L}$ (for a historical résumé of this development, $\rightarrow 238$ ). In most cases, the domain within which these rules and principles were defined was the sentence, although various authors, such as Hjelmslev (1943/1953) or Harris (1952), went beyond the sentence and explored the possibility of defining all and only the well-formed combinations of morphemes within a text or discourse. Experience has taught us, however, that it is the sentence, not the text or discourse, that forms the universe of grammar. There is no valid concept of '(un)grammaticality' for texts or discourses. Instead, texts or discourses are coherent or incoherent, and the principles that make a text or discourse (in)coherent are of a totally 
different nature from those that make sentences (un)grammatical.

Through the works of Bloomfield and Harris, this led to the concept of generative grammar. A generative grammar is a formalism that defines precisely which combinations of morphemes of a language $\mathbf{L}$ form grammatical sentences. This concept of grammar was supported by then recent developments in mathematics, where, during the 1920 s, the notion of algorithm had been extended beyond its traditional boundaries.

Traditionally, an algorithm is a system for the computation of additions, subtractions, multiplications and divisions of numbers. The word algorithm derives from the byname al Huárizmi, "the man from Huárizm" (ancient Chorasmia, now Uzbekistan), of the 9th-century mathematician-astronomer $\mathrm{Mu}-$ hamed ibn Mūsa, who developed our customary ways of doing addition, subtraction, division and multiplication with the help of the Arabic number system including the digit zero. From the 12th century on, this form of arithmetic gradually gained ground, first in Sicily and Southern Spain, then in the rest of Europe, where the term alguarismus (still in Spanish guarismo "number") became algorismus, later garbled into algorithmus, owing to a confusion with the Greek word arithmós 'number'.

During the 1920s mathematicians became aware that the original notion of algorithm has, in fact, a much wider application (see Rosenbloom 1950). The notion was thus extended to cover any formally well-defined system consisting of a finite sequence of mechanical instructions each acting upon input strings of symbols of a certain type and producing output string of symbols. When the input string of symbols is null, the algorithm is a primitive algorithm. Otherwise it is derived. The input to a derived algorithm may itself be characterizable by means of an algorithm, recursively, until a null input is arrived at. But a derived algorithm may also act upon an input that is not algorithmically characterizable, either because of the limited means available at any given time, or in principle.

Thus defined, the notion of algorithm is extremely general (there are at present about six million references to 'algorithm' on the internet). In fact, it became the central notion in computer science. In the more limited perspective of linguistics, however, algorithms are typically used either to characterize a 'language', in the sense of 'set of string of symbols', or to analyze and assign a structure to any input string in the 'alphabet' of some 'language', and thus to decide in a finite number of steps whether the input string does or does not belong to the 'language' in question. The former class of algorithms are generative algorithms, the latter are called decision algorithms or parsers. A 'language' (set of strings of symbols) that can be generated by a primitive algorithm, with or without subsequent derived algorithms, and which can also be parsed by a decision algorithm is called a decidable 'language'. A 'language' that can be generated from null but cannot be parsed by automatic procedure is called a recusively enumerable or canonical 'language'. A 'language' that can neither be generated nor parsed is noncanonical (see Seuren 1998: 267-279). (It is generally assumed among mathematicians that a noncanonical 'language' is chaotic and therefore without interest. Whether this is indeed so, is, however, a moot point. It is not to be excluded that the set of all possible propositional thoughts, though representable as a set of strings of symbols and thus as a 'language', will turn out not to be recursively enumerable and hence to be noncanonical. It seems wiser, therefore, to reserve judgement on the question of whether noncanonical 'languages' are with or without interest.)

As shown in $\rightarrow 217$, the work by Bloomfield and Harris led straight up to a view of grammar as a primitive generative algorithm. This view of grammar has been the hall-mark of generative grammar till the present day. It is implicitly assumed that natural languages are canonical but not decidable. That is, it is assumed that it is possible to generate all and only the sentences of any natural language $\mathbf{L}$ by algorithmic procedure, but the question of whether it is possible to decide, in a finite number of steps, for any given string $\Sigma$ made up of symbols of any natural language $\mathbf{L}$, wether $\Sigma$ is a sentence of $\mathbf{L}$, is left open.

In computational linguistics, by contrast, it is generally assumed that natural languages are decidable, and the algorithms developed in those circles are mostly of the parsing type. It is uncertain whether the more daring position of computational linguistics will turn out to be tenable. So far, all attempts at developing fully automatic parsers have systematically foundered on the fact that in many instances an appeal must be made to users' nonformalized world -or situational knowl- 
edge. No such systematic barrier has been encountered in work on the generative side, where the problems are of a totally different nature. It may well be, therefore, that natural languages are canonical but not decidable. The fact that competence in a language implies the carrying out of parsing procedures on sentences (at some deeply buried nonconscious level of operation) as part of the interpretation process, may well be attributable to the human capacity to draw on all kinds of knowledge as a support for formal computational procedures present in the brain. In natural life, the parsing process would then not be compositional, but co-dependent on support by available knowledge sources. Only when the totality of human knowledge and cognition has been caught in terms of fully formalized algorithmic procedures, will there be sufficient grounds for calling natural languages decidable. So far, however, this ideal is still way beyond our reach. (A similar problem besets work in machine translation.)

An example of a toy system for the primitive algorithmic generation of a few English sentences is the following:

(1) (i) $\mathrm{S} \rightarrow \mathrm{NP}+\mathrm{VP}$

(ii) $\mathrm{NP} \rightarrow \operatorname{Det}(+\mathrm{A})+\mathrm{N}$

(iii) $\mathrm{VP} \rightarrow \mathrm{V}+\mathrm{NP}$

(iv) Det $\rightarrow$ \{the, a\}

(v) $\mathrm{N} \rightarrow$ \{mouse, cat

(vi) $\mathrm{V} \rightarrow$ ate, caught, chased

(vii) $\mathrm{A} \rightarrow$ \{black, big, quick

The start symbol ' $S$ ' of instruction (i) is the null input. The arrows instruct the user (or the machine) to print the symbols to its right, in the order given, underneath the symbol that stands to its left, which has been printed before, and draw connecting lines. Round brackets around a symbol indicate optionality. Curly brackets indicate that one of the elements enclosed must be selected. The symbols to the right of an arrow are the expansion of the symbol to its left. The start symbol ' $S$ ' thus expands into the small bit of tree structure shown in (2a). Further expansions generate, for example, the tree structure (2b), corresponding to the sentence The big cat chased a quick mouse. A system of generative instructions of the expanding type exemplified in (1) is generally known as a Phrase Structure grammar or PS-grammar. PSgrammars generate constituent structure trees. (2a)

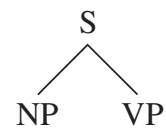

(2b)

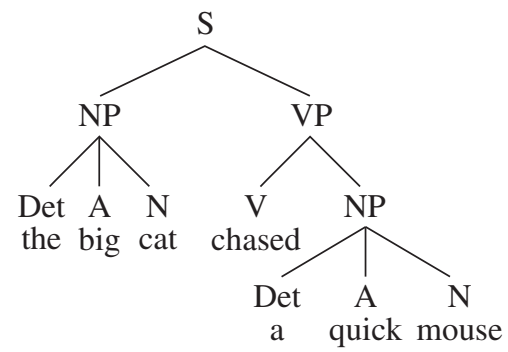

At first, it was hoped that a PS-grammar would do the job of generating all and only the wellformed sentences of a natural language. Harris soon realized, however, in the late $1940 \mathrm{~s}$, that it would not be sensible to attempt to implement such a research program. A more intelligent method would be to exploit the many systematic correspondences between sentence types. For example, active sentences and their corresponding passive forms show systematic structural relations. Likewise for affirmative sentences and their corresponding questions, or positive and negative sentences. It would, therefore, make sense to let a PS-grammar generate the simpler of a pair of related sentence types, and then add a derived algorithm that would produce the other, less simple, sentence type, or would combine several primitive sentence types into one larger, complex one. The sentences generated by the primitive PS-grammar were called kernel sentences. The added derived algorithm was called the transformational component of the grammar, producing transformed sentences. This was the birth of transformational generative grammar or TGG. One may put its birth-date around the year 1950.

Harris (1951) has no notion of transformation yet: its text had been rounded off in 1947, as the Preface tells us. In his subsequent publications, however, starting with 1952 (for a complete collection of the relevant papers, see Harris 1981), the notion gets further developed and more and more refined. The first refinement consisted in dropping the notion of kernel sentence and replacing it with that of deep structure. Instead of letting a PS-grammar generate a fully equipped surface structure, with all the morphological and other trimmings, it seemed 
wiser to let the PS-grammar deliver an unfinished product called 'deep structure' (DS) and feed that into a compulsory system of transformations that would deliver a surface structure one way or another. Thus, for example, a sentence like (3a) would have something like (4a) as its DS. The passive transformation would produce (4b). A common, generalized procedure would then be applied to both (4a) and (4b), yielding (3a) and (3b), respectively.

(3a) The cat has chased the mice.

(3b) The mice have been chased by the cat.

(4a)

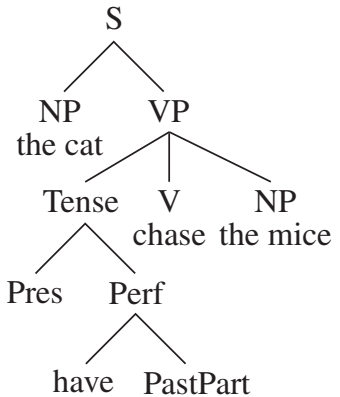

The older 'horizontal' notion of transformation was thus replaced by the newer 'vertical' notion some time during the 1950s. The result was a TGG model of the type shown in Fig. 232.1.

Around that time, the influence of contemporary philosophy of science began to be felt.

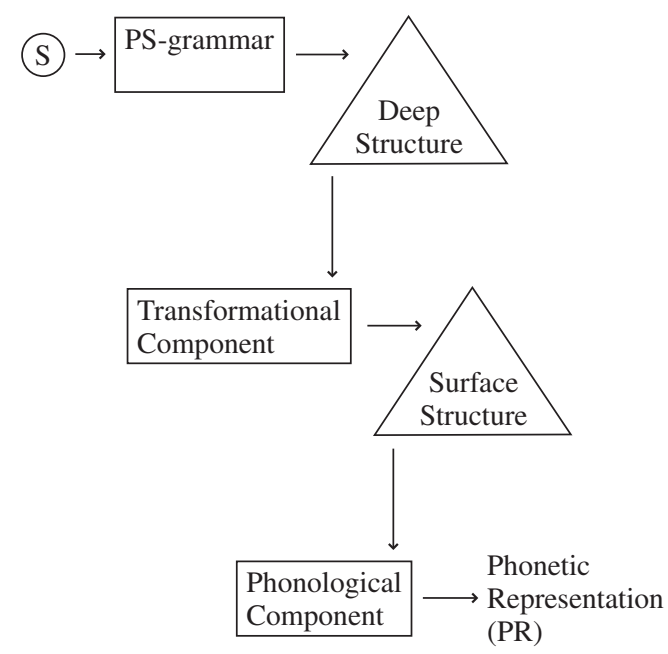

Fig. 232.1: The overall structure of a TGG around 1960
This was rather a new element in the study of language, introduced mainly by Noam Chomsky. According to Chomsky, a generative grammar of a language $\mathbf{L}$ should be seen as a theory of $\boldsymbol{L}$, making explicit the intuitive notion of grammaticality or wellformedness, which enables speakers to distinguish between grammatical and ungrammatical sentences. This speaker's ability was then termed competence in $\boldsymbol{L}$.

Very quickly, however, it began to be observed that this was a little too fast. If a 'theory of $\mathbf{L}$ ' is to make explicit the native speaker's competence in $\mathbf{L}$, it should involve

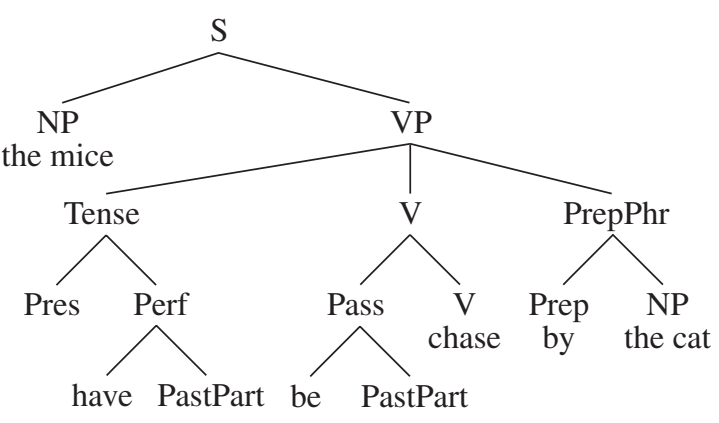

a great deal more than just the ability to distinguish between grammatical and ungrammatical sentences. It makes no sense to say that some being or machine that has just a generative and/or a decision algorithm of $\mathbf{L}$ is competent in $\mathbf{L}$. A competent speaker also knows what the sentences of his language mean. Any theory of $\mathbf{L}$, therefore, should encompass also a formal account of the meanings of the sentences of $\mathbf{L}$. In other words, philosophical reflection on the nature of formal grammars called the semantic chickens home to roost.

\section{The semantic component}

In their 1963 paper, the philosophers Katz and Fodor proposed that a formal algorithmic grammar of the type envisaged should be extended with a semantic component, which should take as input given syntactic structures and deliver something like a semantic analysis (SA) of the sentence at hand. The semantic component would thus be a derived algorithm, meant to give a formally explicit account of the competent speaker's ability to understand and interpret the sentences of his language.

There were, however, some serious difficulties which the 1963 article was unable to 
solve. The first question was how to determine what kind of syntactic structures should be taken as input to the semantic component. Katz \& Fodor proposed that both deep structures (DS) and surface structures (SS) should qualify. Yet their arguments for making that choice were at best flimsy.

The second, even more serious, problem was how to specify the format of the semantic component's output: what does a semantic analysis look like? Here again, Katz \& Fodor failed to provide a satisfactory answer. It was not until McCawley's articles of the late 1960s that this question was unequivocally answered.

Then, the uncertainty regarding the format of SAs inevitably led to a quandary over the format of the algorithmic instructions making up the semantic component. For when it is not known what the output should look like, it cannot be known either what the instructions should amount to. The 1963 article by Katz \& Fodor was thus shrouded in mystery. But it did call attention to the fact that linguistic competence is more than just an ability to separate grammatical from ungrammatical sentences. The Katz \& Fodor 1963 model of a grammar-cum-semantic component is shown in Fig. 232.2.

The first question mentioned above was answered in a book published by the philoso-

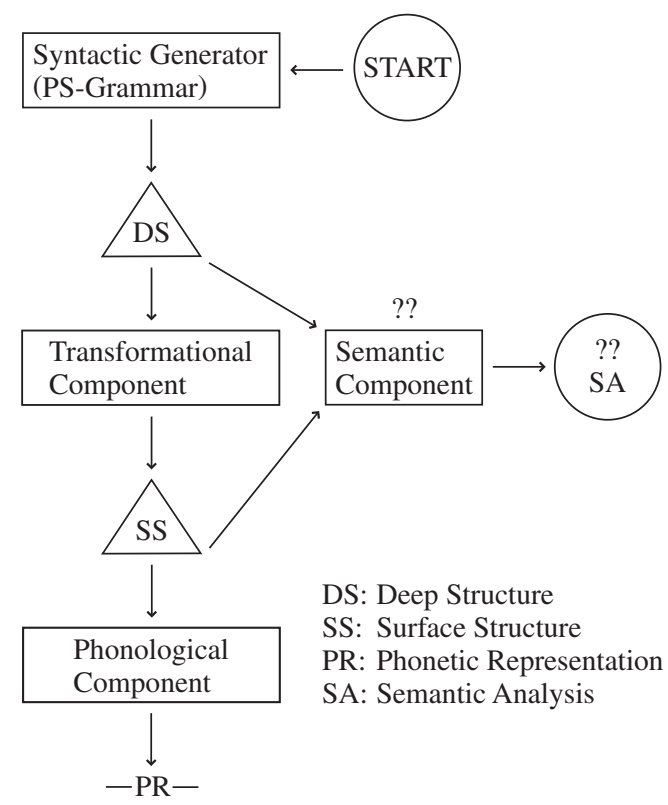

Fig. 232.2: Katz \& Fodor (1963); Chomsky's post1970 'Extended Standard Theory' pher Katz and the linguist Postal in 1964. This book is an extended argument to show that it is only the DS, not the SS, that should be considered the legitimate input to the semantic component. The leading argument was as follows:

If it is assumed that only deep structures are input to the semantic component, so that the transformations cannot have a semantic effect on the sentence under generation, then this assumption will have an overall simplifying effect on the syntax of the language in question.

A few examples will illustrate this. Until 1964 it had been customary among transformationalists to insert the negation (not) transformationally. Katz \& Postal, however, following Klima (1964) where the same proposal is made, point out that there is a range of syntactic phenomena triggered by the negation, including the possible occurrence of negative polarity items (NPIs). If not is inserted transformationally, it will be difficult to provide a unified account of all the syntactic phenomena triggered by it. But if not is part of the DS, then one can, in principle, let it trigger the entire range of phenomena dependent on it in the transformational component. This will unify and simplify the syntactic description of the language. It also means that the semantically highly significant element 'negation' will have found a place in the deep structure, and that, in this respect at least, the transformational component has no semantic effect. (Later studies, e.g., Seuren 1969, showed that the mere presence of not in the DS is not sufficient; its precise scope must also be specified.)

Similar reasoning is applied to the imperative mood. It had been generally assumed among transformationalists that imperatives would have to be generated by the transformational insertion of an element 'IMP', which would then trigger the shaping of the sentence under construction into an imperative form. Katz \& Postal, however, showed that imperatives differ from other sentence types not only in the imperative verb form and the absence of a subject term, but in other respects as well. First, imperatives require an underlying second person subject and an auxiliary will, as appears from reflexivization phenomena and tags, respectively:

(5a) Wash yourself! / *Wash himself!

(5b) Wash yourself, will you! / *Wash yourself, do you! 
Then, certain adverbs cannot occur in imperatives:

(6a) *Perhaps wash yourself! / He will perhaps wash himself.

(6b) *Fortunately wash yourself! / He fortunately washed himself.

The argument is that if the element IMP for 'imperative' is given an appropriate place in the DS of an imperative, together with the requisite second person and the auxiliary will, then the occurrence in imperatives of any offending adverbs can be prevented by ensuring that these adverbs will not co-occur, in DS, with IMP. All the transformations then have to do is assign to the sentence under generation its appropriate surface structure. Transformations are then nothing but merely formal re-arrangements of sentence structures and will not affect meaning.

These and other arguments of a similar nature suggested that, if there should be a semantic component, it should be fed exclusively by DS. A grammar model integrating a semantic component would then be like the model shown in Fig. 232.3, still with the uncertainties regarding the nature of SAs and the format of the instructions in the semantic component. This model, with uncertainties and all, was adopted for a short while by Chomsky, who adhered to it in Aspects (Chomsky 1965).

As regards the remaining uncertainties, James McCawley quickly came to the rescue. In a number of publications McCawley (1967, 1970b, 1972) showed that SAs must themselves be linguistic structures. These should be cast in the mould of the symbolic language current in modern predicate logic a conclusion independently reached in Seuren (1969: 219-224). Moreover, since SAs must themselves be taken to be linguistic structures, they can be represented as constituent trees. This again means that the semantic component's instructions be transformational in nature, since they transform DS tree structures into SA tree structures.

This insight gave rise immediately to the question of the ways in which DSs and SAs could be assumed to differ. One began to wonder what arguments would motivate a distinction between DS and SA levels of representation of sentences. The answer was quick: no arguments were available to maintain that distinction. It was hence concluded that these two levels of representation should

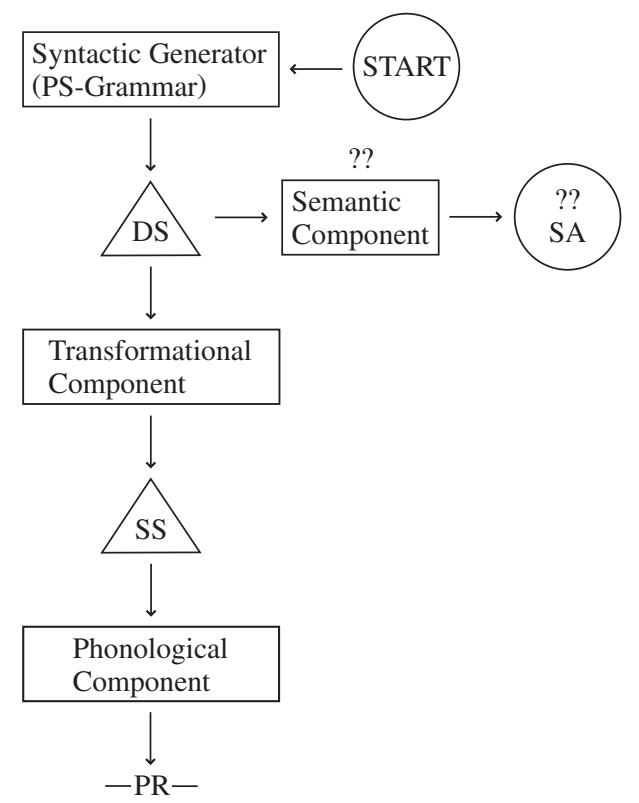

Fig. 232.3: Katz \& Postal (1964) and Chomsky (1965)

be collapsed. What had been taken to be the form of DS representations should at the same time be reckoned to be the SA of any sentence under generation. Exit the semantic component.

The collapsing of DS and SA was revolutionary in many ways, not all of which could be suspected at the time. The general feeling at the time was that, finally, syntax and semantics were being united again, as they had been during the whole history of linguistics till the advent of structuralism and behaviorism in the early years of the 20th century. As from 1965, a new movement arose, which was soon called: Generative Semantics. Its main protagonists were the linguists James McCawley, George Lakoff, John Robert ('Haj') Ross, and Paul Postal, and the philosopher Jerrold Katz, with lateral support from Jerry Fodor and a few other philosophers. In a couple of years' time generative semantics swept the linguistic world. Yet it maintained its overall dominant position for no more than a decade: around 1975 a conservative counterrevolution, led by Noam Chomsky, had regained dominance and generative semantics was declared dead. It would take too long to describe and analyze this curious and historically unique development in detail here. (See Seuren 1998: 493-527, for an extensive discussion.) Unfortunately, New- 
meyer (1980) is not reliable, as shown in McCawley (1980); its second edition of 1986 fares no better. Suffice it to quote from Huck \& Goldsmith's admirable study (1995: ix):

But while the Generative Semanticists unquestionably faced theoretical obstacles of various sorts, there are also good reasons to believe that the demise of their program was not a consequence of theoretical weakness. Indeed we will argue in what follows that it is not possible to find, internal to the idea of Generative Semantics as it was evidently originally understood by Lakoff, McCawley, Postal and Ross, adequate grounds to explain its widespread abandonment in the 1970s. We will be concerned to evaluate the linguistic evidence on its own terms, paying particular attention to the theoretical assumptions that underlay the various critiques, and will conclude that one must turn to external explanations to account adequately for what transpired. But although external factors undoubtedly affected the way that the various proposals in the dispute were understood and received, we would also suggest that a focus on the relatively dramatic personal and social aspects of the interactions in which the participants were involved has tended to obscure the conceptual significance of the positions they took.

Be all that as it may, it must be emphasized that the ideas that came to the fore in the context of generative semantics implied a radical departure from the well-established overall parameters that had been taken to define the notion of grammar since Bloomfield (1933), Harris (1951), and Chomsky (1957). In the established view, a grammar is an algorithmically organized random sentence generator meant to characterize precisely the set of well-formed sentences of a language. Let us call this the random-generator of grammar. By contrast, generative semantics was quickly leading to a view in which a grammar is a transformational device mediating between cognitively produced thoughts on the one hand and well-formed linguistic surface structures on the other. Let us call this the mediational view of grammar.

Speaking in realistic terms of actual cognitive processing, the mediational view places the infinitely creative power of a language not in the language itself, as the random-generator view does, but in cognition. The fact that a language must be treated as an infinite set of possible sentences is not denied in the mediational view. What is denied is that the cause of the infinity of language must be sought in language itself. In the mediational view, the cause of the infinity of language lies in the fact that cognition is able to produce an infinite variety of different propositional thoughts. All language has to do is make sure that each such product is expressed in a natural language sentence. And clearly, in order to do that, language needs a machinery that allows for infinitely many different combinations of morphemes into sentences (and also for infinitely many different combinations of sentences into texts). The development of generative semantics thus announced a radical paradigm shift in linguistics, from a random-generator to a mediational perspective. As from 1970, Chomsky did all he could to prevent such a paradigm shift, in which he was not playing a leading role, from happening.

But let us revert to the details of the development. The collapsing of DS and SA naturally placed the syntactic generator or PSgrammar in a completely different light. A PS-grammar now became a system defining or producing structures belonging to predicate logic and it somehow looked very odd to regard such a system as a random sentence generator. It was much more natural to establish a link with cognition and interpret the DS/SA structures as the products of so far unexplored and largely mysterious thought processes, thereby reinforcing the realist interpretation of the model. Yet many felt that it was too hazardous, in a period when behaviorism was still on everybody's mind, to speak openly of 'thoughts' and 'cognitive processes'. Noncommittal talk of 'logic' was a great deal more secure. Even so, it was universally felt that these new ideas were bringing the structural study of language closer to the study of cognition and that this meant a more organic integration of linguistics into the wider group of human sciences as well as a return to tradition.

In this context it was both relevant and opportune to drop the notion of a PS-grammar as a random algorithmic syntactic generator and redefine a PS-grammar as a set of structural constraints on the form of the constituent trees embodying the DS/SA representations. This idea was formally elaborated in McCawley (1968). (One also notes the opening sentence in McCawley (1970a): "The contents of this paper is clearly transformational grammar but not so clearly generative grammar.") If only one had the courage to venture the leap to cognition, a PS-grammar could be regarded as a mere corollary to the overall cognitive procedure of casting 
(7a)

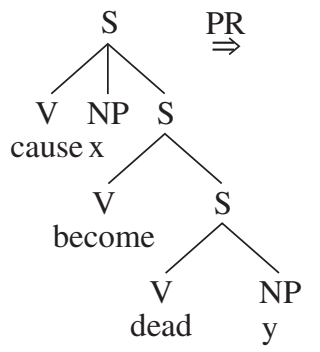

(7b)

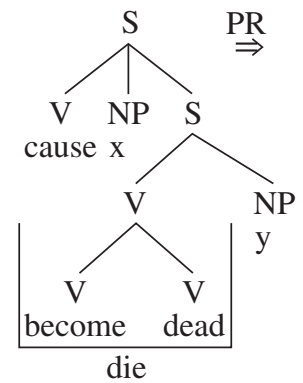

(7c)

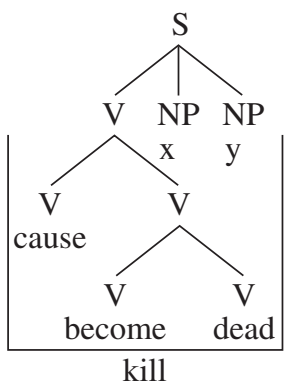

thoughts into the format of DS/SA representations.

This leap was never taken by McCawley, who stayed within the boundaries of logic, no matter how ill-defined the relation of logic was with regard to cognition. Others, however, did venture the leap. Katz, for example, wrote (1966: 98):

Roughly, linguistic communication consists in the production of some external, publicly observable, acoustic phenomenon whose phonetic and syntactic structure encodes a speaker's inner, private thoughts or ideas and the decoding of the phonetic and syntactic structure exhibited in such a physical phenomenon by other speakers in the form of an inner, private experience of the same thoughts or ideas. Behavioristically oriented investigations of linguistic communication focus exclusively on the publicly observable aspects of communication situations: speech sounds, nonverbal behavior or the participants in the situation, and physical properties of available stimuli. Thus, such investigations neglect the essential aspect of successful linguistic communication, the congruence of speaker's and hearer's thoughts and ideas that results from verbal exchanges.

Fodor (1976) went further, trying to be more definite on the question of what thoughts are made of. Reviving certain Ancient and Medieval views, he proposed that what we may reasonably call 'thought' is also a 'language' of some special kind (Fodor 1976: 68):

One way of describing my views is that organisms (or, in any event, organisms that behave) have not only such natural languages as they may happen to have, but also a private language in which they carry out the computations that underlie their behavior. I think this is a fair characterization of what I have been saying, but I recognize that some philosophers would take it to be a reductio ad absurdum argument.

In this view, a grammar is indeed a transducer taking thought structures as input and delivering surface structures as output. But even for those who did not, or not fully, accept Fodor's position on the nature of thought, it was becoming clear that the input to the grammar machinery of a natural language should be taken to be a mental product created by so far unknown or badly understood processes of thought.

Great store was set, at the time, by what was called prelexical syntax, the internal analysis of semantically complex predicatives. Following proposals made by McCawley, there was a sudden rash of 'prelexical' analyses. McCawley had proposed, in McCawley (1967) and other papers, that the English verb kill should be analysed as 'cause-to-become-dead'. This analysis would then be subject to an internal cyclic transformation called Predicate Raising (PR), which would yield a V-cluster that would be a candidate for lexicalisation as kill. The procedure is illustrated in $(7 a)-(7 c)$.

One of the claims of prelexical syntax was that the internal lexical processes of unification under a single categorial node $(\mathrm{V})$ were identical to transformational processes found in the 'open' syntax of languages. This was denied by Chomsky, who claimed (1972: 142) that the rule of Predicate Raising lacked empirical support: "... the unit that is replaced by kill is not a constituent, but it becomes one by the otherwise quite unnecessary rule of predicate raising." It was then found, however (Seuren 1972; Evers 1975) that Chomsky was wrong, as French, Italian, Dutch, German, Japanese, Turkish and many other languages have PR as a strongly supported rule in their 'open' syntax.

Prelexical syntax also claimed that the transformational processes of prelexical and 'open' syntax should be taken to be intermingled: McCawley-type prelexical syntax denied that any 'deep structure' level should be assumed at which all surface lexical items have 


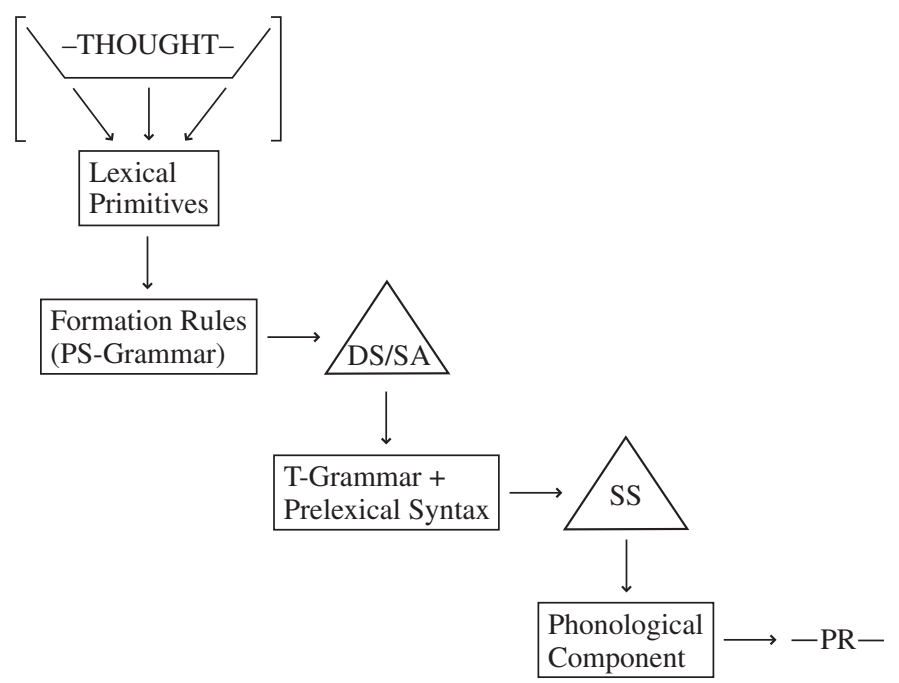

Fig. 232.4: The Generative Semantics model $( \pm 1970)$

been filled in. The only 'deep' level of representation, SA, was said to have only 'primitive' lexical items filled in. The surface items were taken to be as much the result of transformational processes as the rest of surface structure. This second proposal has not carried the day, mainly because it became clear that prelexical analyses of the kind exemplified in $(7 a)-(7 c)$ fail to do full semantic justice to the replacing surface lexical items. It is now widely accepted that, although prelexical syntax probably makes a great deal of sense, it whould not be taken to be a part of 'open' syntax. It is, therefore, advisible to assume that any semantic level of representation is, in principle, equipped with the surface lexical items of the sentence in question. Any further 'internal' analysis should be relegated to the lexicon.

The overall model of grammar adopted by the generative semanticists thus looks as in Fig. 232.4. The 'THOUGHT' section at the top of the diagram has been placed between square brackets to indicate that this part is not even close to a formal treatment yet and must, for the moment, be taken at face value.

As has been said above, generative semantics was overrun by Chomskyan grammar during the first half of the 1970s. After 1975 none of the protagonists continued to work according to the research program inherent in generative semantics. The only linguist who has steadfastly held on to the mediational paradigm as it resulted from generative semantics, renaming it Semantic Syntax, has been Seuren, in a series of publications till the present day (see in particular Seuren 1996).

But what happened in the main theater of theoretical linguistics? During the late 1960s Chomsky realized that his 1965 position, which was in principle that of Katz \& Postal (1964), shown in Fig. 255.3 above, was untenable and would, if maintained, inevitably lead to the generative semantics position as it was being developed and promoted by a group of new protagonists. Since this was in any case unacceptable, he had to change his position. The change was, however, presented as a further development. Having called the 1965 position the 'standard theory', he gave the name 'extended standard theory' or EST to his new position (Chomsky 1972). The extension, however, consisted, in fact, in a reversal to the Katz \& Fodor position of 1963 depicted in Fig. 232.2 above.

In contrast to Katz \& Fodor (1963), however, Chomsky did produce arguments to support the thesis that semantic interpretation is a question not only of deep structure but also of surface structure. The semantic elements that, according to him, should be derived from the surface structure rather than from the deep structure of a sentence were of two kinds: (a) contrastive accent and/ or topic-focus modulation and (b) scope differences corresponding to the linear order of scope-bearing semantic operators.

As regards contrastive accent and/or topicfocus modulation, Chomsky's argument was (1972: 99-100): 
Rules of phonological interpretation assign an intonational contour to surface structures. Certain phrases of the surface structure may be marked, by grammatical processes of a poorly understood sort, as receiving expressive or contrastive stress, and these markings also affect the operation of the rules of phonological interpretation. If no such processes have applied, the rules assign the normal intonation. In any event, phrases that contain the intonation center may be interpreted as focus of utterance, the condition being perhaps somewhat different and more restrictive when the intonation center involves expressive or contrastive stress, as noted.

This, however, did not take into account the fact that contrastive or focus accent is sometimes impossible and sometimes obligatory, in both cases under semantically controlled conditions. Such facts inevitably put paid to the contention that contrastive or focus accent is a matter of surface structure interpretation.

Consider, for example, (8a) and (8b) (Seuren 1974: 187):

(8a) I don't believe Tom has yet seen the problem.

(8b) *I don't believe Tom has yet seen the problem, Fred does.

Although (8a) is a grammatical English sentence, $(8 \mathrm{~b})$ is not. The reason for the ungrammaticality of ( $8 b)$ is clearly semantic, since (8b) corresponds to the semantically more explicit but equally ungrammatical "*the one who believes that Tom has yet seen the problem is not me but Fred'. Clearly, the negative polarity item yet is the offending element: it requires a negation within the believe-clause, not outside it and over the cleft construction that corresponds with the contrastive accent.

On the other hand, contrastive accent is obligatory in a sentence like (9a). The corresponding (9b), without the contrastive (focus) accent, is ungrammatical as it requires the reflexive myself:

(9a) I didn't teach me, YOU did.

(9b) *I didn't teach me.

Again, the reason is to do with the semantic analysis of (9a), which corresponds to 'the one who taught me is not me but you', which is well-formed and does not require reflexivization of me. Without the cleft construction, reflexivization is obligatory.

As regards operator scope differences as expressed in the linear order of their surface representatives, it had been noted by a vari- ety of authors (e.g., Jackendoff 1969, Seuren 1969, Lakoff 1971, McCawley 1972) that, as a matter of principle though not as an absolute rule, higher scope operators precede lower scope operators in surface structure, as is shown by the following examples:

(10a) Nobody here knows two languages.

(10b) Two languages are known by nobody here.

In most academic surroundings, (10a) will be false while (10b) will be true. The difference lies in the different scopes of the underlying logical operators NOT, SOMEONE, and TWO. In the normal interpretation of (10a) the logicosemantic order is NOT-SOMEONE-TWO, whereas (10b) is normally interpreted with the order Two-NOT-SOMEONE. Many more such examples could, of course, be given.

Following Jackendoff (1969), Chomsky maintains that "the scope of negation will be determined by the position of not in surface structure" (1972: 104), extending this to other logical operators as well. The point is, however, that the scope-order correspondence (if taken to be an absolute principle) works both ways. In sentence comprehension, the listener will take the surface structure as point of departure and reconstruct the logico-semantic scope of whatever scope-sensitive operators occur in a sentence on the basis of their linear order. In sentence production, however, the logico-semantic structure, with scope expressed in the SA as a command relation, is cast into a surface structure with the operators ordered linearly according to their scope. Clearly, if one takes a generative point of view with regard to an algorithmical grammar, the latter arrangement, from SA to SS, is the more natural one.

Proposals as to how to relate SA-scope with linear order in SS were made in Seuren (1969), Lakoff (1971) and McCawley (1972; in circulation since 1969). These proposals amounted to a transformational treatment in terms of a rule or process of Operator Lowering, according to which an operator, once lowered into the matrix-S, was 'flagged', so that any subsequent operator would have to stay on its left, if allowed to do so by the rest of the grammar. That the grammar does not always allow this, appears from the following example:

(11a) German:

Ich verstand ein Wort nicht.

I understood one word not ONE NOT 
(11b) English:

*I understood one word not.

Sentence (11a) is perfectly good German and is unambiguously interpreted as saying that there is one word which the speaker failed to understand. The scope-ordering constraint requires ONE to precede NOT in surface structure, which is possible in German, where the negation is lowered to the far right of the matrix-S, unless stopped by a previously lowered operator, as in:

(12) German:

$$
\begin{array}{lll}
\text { Ich verstand kein } & \text { Wort. } \\
\text { I understood not-a } & \text { word } \\
& & \text { NOT ONE }
\end{array}
$$

In English, however, not is lowered onto the verb of the matrix-S (normally with $d o$-support), or onto a following existential or a preceding existential or universal quantifier.

(13a) I found no mistake.

(13b) No mistake escaped him.

(13c) *I found not all mistakes.

(13d) Not all mistakes were found.

Given these restrictions on the lowering of not in English, it follows that (11b) is ungrammatical and its semantic content must be expressed by different means.

Apart from such complications, however, one may consider the possibility that the scope-order correspondence of logico-semantic operators can be handled either way: from SA to SS or vice versa. In fact, May (1977) proposed a machinery, entirely in the spirit of Chomsky's EST, to raise operators from SS to their position in the corresponding SA (called 'logical form' by the Chomskyans). The machinery proposed was a simple reversal of the lowering machinery proposed by Lakoff, McCawley and others, yet no attribution was made to the generative semantics literature on the subject, a curious omission that caused some surprise at the time.

The EST position was, therefore, not very strong. Around 1980 Chomsky changed position again, now in a direction as far away from generative semantics as possible: semantic interpretation was now considered to be grafted onto SS exclusively, to the exclusion of DS, as shown in Fig. 232.5. The new theory went under a variety of names, here summarized as the 'post-EST position'. It was in force from roughly 1980 till 1995, when Chomsky's Minimalism became official.

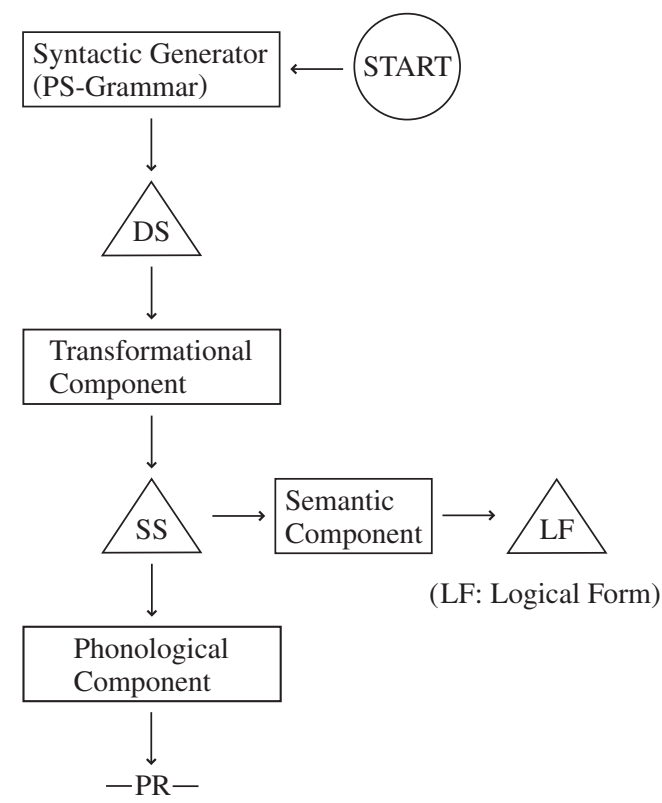

Fig.: 232.5: Chomskyan post-EST grammar $( \pm 1980$ till \pm 1995$)$

Even before EST passed into post-EST, McCawley's argument that SAs should be regarded as linguistic structures cast into the language of modern predicate logic had become accepted, but, again, without any attribution. The term 'logical form' (LF) was introduced to stand in for 'semantic analysis' or 'semantic representation'.

Again, however, the post-EST position turned out to be unstable. For it turned out to be systematically the case that DS and LF were either identical or so close to each other that the distinction between the transformational and the semantic component, and hence between DS and LF, became highly questionable. An appeal to the different 'directionality' of either component was ruled out, as Chomsky had ruled in (1972) that directionality of rule systems was just a notational matter.

An example will illustrate this. Consider the sentence (14a):

(14a) John is likely to win.

(14b) $\mathrm{s}\left[\mathrm{s}\left[\right.\right.$ John - win] ${ }_{\mathrm{VP}}[$ be likely $\left.]\right]$

This would be transformationally derived from an underlying DS roughly corresponding to (14b), meaning 'that John win is likely'. However, that is also what the sentence means, so that the same (14b) is not 
2212 XXXIV. The Development of Theories of Semantics, of the Lexicon, and Semantic-Based Theories

only the underlying syntactic structure but also the resulting LF. It thus looked as if the old argument raised by the generative semanticists kept haunting the random-generator linguists.

In 1995 Chomsky published his book The MinimalistProgram, where the position is changed again. It would take us too far to go into the details of how 'logical form' is treated there (see Seuren 2004 for a critique). In the present context it should be sufficient to refer to Johnson \& Lappin (1997), who point out that the minimalist notion of 'logical form' is entirely undefined and, to the extent that it can be reconstructed, unintelligible. It would seem, therefore, that the latest version of the Chomskyan random-generator approach to grammar and meaning is farther removed than ever from a satisfactory conclusion.

\section{Bibliography}

Chomsky, Noam. 1957. Syntactic Structures. (= Janua Linguarum, 4.) The Hague: Mouton.

Chomsky, Noam. 1965. Aspects of the Theory of Syntax. Cambridge Mass.: MIT Press.

Chomsky, Noam. 1972. Studies on Semantics in Generative Grammar. (= Janua Linguarum, 107.) The Hague: Mouton.

Chomsky, Noam. 1995. The Minimalist Program. Cambridge, Mass.: MIT Press.

Evers, Arnold. 1975. The Transformational Cycle in Dutch and German. PhD thesis, Utrecht University. (Reproduced by Indiana University Linguistics Club, Bloomington.)

Fodor, Jerry A. 1976. The Language of Thought. Hassocks, Sussex: Harvester Press.

Harris, Zellig S. 1951. Methods in Structural Linguistics. Chicago: University of Chicago Press.

Harris, Zellig S. 1952. "Discourse Analysis". Language 28:1.1-30. (Also in Harris 1981: 107-142.)

Harris, Zellig S. 1981. Papers on Syntax. Ed. by Henry Hiž. Dordrecht: Reidel.

Hjelmslev, Louis. 1953 [1943]: Prolegomena to a Theory of Language. Translated from the Danish by Francis J. Whitfield. (= Indiana University Publications in Anthropology and Linguistics; Memoir 7 of $I J A L$.) Baltimore, Md: Waverly Press.

Huck, Geoffrey J. \& John A. Goldsmith. 1995. Ideology and Linguistic Theory. Noam Chomsky and the deep structure debate. London \& New York: Routledge.
Jackendoff, Ray S. 1969. “An Interpretative Theory of Negation". Foundations of Language 5.218-241.

Johnson, David E. \& Shalom Lappin. 1997. "A critique of the Minimalist Program". Linguistics and Philosophy 20.273-333.

Katz, Jerrold J. 1966. The Philosophy of Language. New York: Harper \& Row.

Katz, Jerrold J. \& Jerry A. Fodor. 1963. "The Structure of a Semantic Theory". Language 39: $2.170-210$.

Katz, Jerrold J. \& Paul M. Postal. 1964. An Integrated Theory of Linguistic Descriptions. Cambridge, Mass.: MIT Press.

Klima, Ed. 1964. "Negation in English". The Structure of Language: Readings in the philosophy of language ed. by Jerry A. Fodor \& Jerrold J. Katz, 246-323. Englewood Cliffs, N. J.: PrenticeHall.

Lakoff, George. 1971. "On Generative Semantics". Steinberg \& Jakobovits, eds. 1971. 232-296.

May, Robert. 1977. The Grammar of Quantification. PhD thesis, MIT, Cambridge, Mass.

McCawley, James D. 1967. "Meaning and the Description of Languages". Kotoba no Uchu 2: 9.1018; 10.38-48; 11.51-57. (Repr. in McCawley 1973. 99-120.)

McCawley, James D. 1968. "Concerning the Base Component of a Transformational Grammar". Foundations of Language 4.243-269.

McCawley, James D. 1970a. "Where Do Noun Phrases Come from?". Readings in English Transformational Grammar ed. by Roderick A. Jacobs \& Peter S. Rosenbaum, 166-183. Waltham, Mass.: Ginn \& Co. (Repr. in McCawley 1973. 133-154, and in Steinberg \& Jakobovits 1971. 217-231.)

McCawley, James D. 1970b. "Semantic Representation". Cognition: A multiple view ed. by Paul L. Garvin, 227-247. New York: Spartan Books. (Repr. in McCawley 1973. 240-256.)

McCawley, James D. 1972. "A Program for Logic. Semantics of Natural Language ed. by Donald Davidson \& Gilbert Harman, 498-544. Dordrecht: Reidel. (Repr. in McCawley 1973. 285-319.)

McCawley, James D. 1973. Grammar and Meaning. Papers on syntactic and semantic topics. Tokyo: Taishukan.

McCawley, James D. 1980. Review of Newmeyer (1980). Linguistics 18: 9/10.911-930.

Rosenbloom, Paul C. 1950. The Elements of Mathematical Logic. New York: Dover.

Newmeyer, Frederick J. 1980. Linguistic Theory in America: The first quarter-century of Transforma- 
tional Generative Grammar. New York, London, etc.: Academic Press. (2nd rev. ed., 1986.)

Seuren, Pieter A. M. 1969. Operators and Nucleus: A contribution to the theory of grammar. Cambridge: Cambridge University Press.

Seuren, Pieter A. M. 1972. "Predicate Raising and Dative in French and Sundry Languages. Paper, Magdalen College, Oxford. (Printed in Seuren 2001, Chap. 7.)

Seuren, Pieter A. M. 1974. "Negative's Travels". Semantic Syntax ed. by P. A. M. Seuren, 183-208. Oxford: Oxford University Press.

Seuren, Pieter A. M. 1996. Semantic Syntax. Oxford: Blackwell.
Seuren, Pieter A. M. 1998. Western Linguistics: An historical introduction. Oxford: Blackwell.

Seuren, Pieter A. M. 2001. A View of Language: Collected papers 1972-1999. Oxford: Oxford University Press.

Seuren, Pieter A. M. 2004. Chomsky's Minimalism. New York: Oxford University Press.

Steinberg, Danny D. \& Leon A. Jakobovits, eds. 1971. Semantics: An interdisciplinary reader in philosophy, linguistics and psychology. Cambridge: Cambridge University Press.

Pieter Seuren, Nijmegen (The Netherlands)

\section{Semantic theories in 20th-century America: An overview of the different approaches outside of generative grammar}

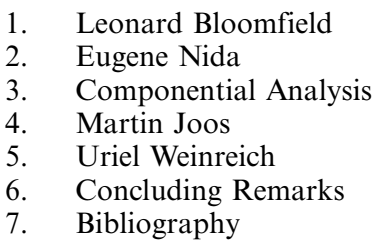

\section{Leonard Bloomfield (1887-1949)}

\subsection{Introduction}

Indigenous and European influences came together, most notably through the work of Noah Webster (1758-1843), William Dwight Whitney (1827-1894), and Ferdinand de Saussure (1857-1913) to shape 20th-century linguistics in America. Alongside the already rich and diverse philological traditions represented by these scholars, anthropology had already exerted its influence through Franz Boas (1858-1942) and Edward Sapir (18841939) when The Linguistic Society of America was formed in 1924, under the presidency of Leonard Bloomfield. The behavioral psychology of John B. Watson (1878-1958) took root in the $1920 \mathrm{~s}$, and had become so potent a force by the 1940s that it played a role in the profound misunderstanding over Bloomfield's views on the place of meaning in linguistics. Believing that he advocated the study of language without meaning, linguists committed themselves to this position. Bloomfield himself decried this as a trend that would "injure the progress of our science by setting up a fictitious contrast between students who consider meaning and students who neglect or ignore it. The latter class, so far as I know, does not exist" (Fries 1954: 160). Charles Carpenter Fries (18871967) attempts to set the record straight, and Charles F. Hockett, Bloomfield's chief disciple, makes an unconvincing argument in defence of his mentor's position (Hockett 1968: 22ff.), but neither provides a comprehensive perspective of Bloomfield on meaning. The need for such a perspective becomes apparent when we recall that from his early studies at the universities of Göttingen and Leipzig prior to World War I, Bloomfield had learned the methodology of the Junggrammatiker and contemporary psychology. This training accounts for the substantial emphasis that Bloomfield gave to historical linguistics - an emphasis that came to be balanced by a synchronic approach to the study of language, under the influence of Saussure.

\subsection{Bloomfield's Definition of Meaning}

No misprision of Bloomfield's thought would have arisen unless he had, albeit unintentionally, created the impression of shunning meaning. How then? By excluding the use of meaning from the method of analysis and deferring the treatment of meaning as an object of analysis. Consequently, Fries (1954: 58), like Hockett (1968), is unable to buttress his defence with explicit references from Bloomfield's writings, quoting instead from Zellig 